Instituto Internacional de Investigación y Desarrollo Tecnológico Educativo INDTEC, C.A.

DOI: https://doi.org/10.29394/Scientific.issn.2542-2987.2018.3.8.13.248-271

OAI-PMH: http://www.indteca.com/ojs/index.php/Revista Scientific/oai

\title{
Inteligencias que Desarrolla el Estudiante Jugador de Ajedrez
}

Autoras: Yuraima Margelis Matos De Rojas Universidad Nacional Experimental Simón Rodríguez, UNESR yuraimatos01@gmail.com

Maryelin Lorena Vethencourt Godoy Universidad Nacional Experimental "Rafael María Baralt", UNERMB mayerajedrez@gmail.com Magda Lisbeth Peña Briceño Universidad Nacional Experimental "Rafael María Baralt", UNERMB bmagdaunica@hotmail.com Magda Violeta Briceño Universidad Nacional Experimental "Rafael María Baralt", UNERMB bmagda@hotmail.com.ar Trujillo, Venezuela

\section{Resumen}

Para fortalecer el desarrollo cognitivo en los estudiantes se requiere el uso de estrategias innovadoras, creativas y formativas que le permitan lograrlo, siendo una de las estrategias didácticas el ajedrez. Por lo que se planteó como propósito de investigación: Identificar las inteligencias que desarrolla el estudiante atleta de la Unidad Educativa de Talentos Deportivos que juegan ajedrez, para sugerir algunas recomendaciones que puedan ser puesta en práctica en las instituciones educativas. Metodológicamente se abordó desde el paradigma cualitativo a través de método fenomenológico que permite develar la realidad desde las experiencias y vivencias de los actores sociales. Constituyéndose como actores clave seis estudiantes de dicha institución jugadores de ajedrez, a quienes se les aplicó una entrevista abierta para obtener la información necesaria, la cual se sistematizó para extraer las categorías, codificaciones y triangular la información. Como resultados se obtuvo que los estudiantes desarrollan las inteligencias: lógica-matemática, la lingüística, la espacial y visual, como la intrapersonal durante el juego de ajedrez y en los procesos de aprendizaje. Configuradas en categorías, analizadas e interpretadas desde las voces de los actores sociales, teóricos e investigadores. Sugiriendo algunas recomendaciones que pueden ser puesta en práctica para fortalecer las inteligencias en el estudiante.

Palabras clave: inteligencia; método de enseñanza; juego educativo. 


\title{
Intelligences Developed by the Student Chess Player
}

\begin{abstract}
To strengthen cognitive development in students requires the use of innovative, creative and formative strategies that allow it to achieve, being one of the didactic strategies chess. For what was proposed as research purpose: Identify the intelligences developed by the student athlete of the Sports Talent Education Unit that play chess, to suggest some recommendations that can be put into practice in educational institutions. Methodologically it was approached from the qualitative paradigm through a phenomenological method that reveals the reality from the experiences and experiences of the social actors. Six key students of the institution were chess players, to whom an open interview was applied to obtain the necessary information, which was systematized to extract the categories, codifications and triangulate the information. As results, it was obtained that the students develop the intelligences: logical-mathematical, linguistic, spatial and visual, as the intrapersonal during the game of chess and in the learning processes. Configured in categories, analyzed and interpreted from the voices of social actors, theorists and researchers. Suggesting some recommendations that can be put into practice to strengthen the intelligences in the student.
\end{abstract}

Keywords: intelligence; teaching method; educational game.

Date Received: 21-11-2017

Date Acceptance: 22-01-2018 


\section{Introducción}

En los últimos años, se viene haciendo énfasis en la necesidad de una educación de calidad, donde se fortalezcan aprendizajes creativos, innovadores, constructivistas, colaborativos, motivadores y, significativos. Aprendizajes que lleven a construir conocimientos, al desarrollo de habilidades y destrezas útiles para la vida. Parar ello, se hace necesario hacer uso de estrategias o recursos pedagógicos que contribuyan al desarrollo de las habilidades intelectuales del estudiante, que lo lleven a resolver problemas, elaborar propuestas y buscar soluciones en cualquier contexto en el que se desenvuelva.

En este sentido, se trata de propiciar el desarrollo cognitivo del estudiante, por lo que el ajedrez, viene a constituir una estrategia, tal como lo refiere Blanco (2007a): "para organizar la esfera cognitiva del ser humano; es decir, el ajedrez permite desarrollar habilidades para resolver situaciones problemáticas, realizar conjeturas, comprobar hipótesis y plantear nuevos problemas" (pág. 81). Por tal motivo, el ajedrez tiene un marcado carácter educativo sobre la personalidad del ser humano, en particular en los estudiantes, quienes en su accionar educativo pueden demostrar sus habilidades en las diferentes situaciones que se le presenten.

Desde esta perspectiva, se puede señalar que a través de la práctica de ajedrez se pueden desarrollar las inteligencias del educando, utilizando esta disciplina deportiva como estrategia pedagógica en el proceso de enseñanza-aprendizaje. Para Blanco, Fernández, Mayor y Martos (2010): el ajedrez puede ser una excelente herramienta para educar y mejorar la inteligencia emocional de quienes la practican.

Cabe referir que las inteligencias de acuerdo con Gardner (citado en López, 2006a): son "la capacidad para resolver problemas y crear productos que son de importancia en el contexto cultural o en una comuna determinada" (pág. 9). En este sentido, se tiene como premisa, valorar la educación a partir 
de reconocer que en el proceso educativo se debe fortalecer la inteligencia como capacidad que se desarrolla al ser estimulada mediante estrategias cognitivas. Autor que propone ocho inteligencias múltiples que el ser humano posee, localizadas en diferentes regiones del cerebro, las cuales son: LógicaMatemática, Verbal-Lingüística, Visual-Espacial, Musical, Corporal, Intrapersonal, Interpersonal, y la Naturalista.

Con ello, se reconoce la existencia de diversas inteligencias, que pueden interactuar y potenciarse entre sí. Para López (2006b): "las inteligencias múltiples no funcionan aisladamente, cualquier rol social que se asuma requiere una combinación de habilidades; porque las inteligencias trabajan juntas para resolver situaciones" (pág. 11). Siendo necesario que los docentes se apropien de diversas estrategias para propiciar su desarrollo.

Por lo que Blanco (2007b): indica "en investigaciones educativas, una de las estrategias que contribuye al desarrollo de las inteligencias es el ajedrez, así como a ejercitar cualidades personales de cada individuo, a la vez le ayuda a resolver problemas personales y de conducta" (pág. 28). En este escenario, Pérez (2012): ratifica que la práctica del ajedrez permite ejercitar la mente debido a la combinación de procesos cognitivos, llevando a un mejor desenvolvimiento de las personas, potenciando sus habilidades y destrezas en la toma de decisiones.

Sin embargo, Blanco (2007c): señala que "en el proceso educativo la práctica del ajedrez es vista como limitaciones enmarcadas en viejos esquemas, por lo general asocian el ajedrez al lograr académicamente procesos lógicos-matemáticos reclinado en el razonamiento humano" (pág. 58). A pesar de la relevancia del juego de ajedrez como estrategia para el desarrollo de las inteligencias en los estudiantes, y que ha sido propuesto por el Ministerio del Poder Popular para la Educación desde los primeros niveles educativos, aún se sigue observando en diferentes instituciones educativas, que la mayoría de las escuelas y liceos no lo utilizan como estrategia para el 
desarrollo de las habilidades cognitivas y, menos, para el desarrollo de la inteligencia en el estudiante.

Apreciación, que se confirma con el estudio de Altuve (2015): quien realizó una investigación en la Unidad Educativa Básica Pre vocacional Agua Clara, donde plantea que el ajedrez no se aplica como estrategia ecológica para el desarrollo de las inteligencias múltiples en los estudiantes de educación primaria, desaprovechando así los beneficios que ofrece como estrategia innovadora, creativa y transformadora.

Desde lo planteado, se hizo necesario situar a la Unidad Educativa de Talentos Deportivos ubicada en el sector San Luis Parte Baja Avenida La Feria del municipio Valera, donde los estudiantes de dicha institución practican diferentes deportes; considerando para el estudio a los estudiantes atletas que practican el ajedrez, y así, identificar las inteligencias que manifiestan cuando juegan el ajedrez y su puesta en práctica en las diversas áreas del conocimiento.

En tal sentido, se planteó como propósito de investigación: Identificar las inteligencias que desarrolla el estudiante atleta al jugar ajedrez, de manera que permita sugerir algunas recomendaciones que puedan ser puesta en práctica en las instituciones educativas, y así fortalecer el desarrollo de las inteligencias al relacionarlas con las diferentes áreas del saber. Estudio que se inserta en la Línea de Investigación Investigadores en Acción Social, IAS, UNESR, Núcleo Valera.

\section{Fundamentos teóricos}

Como fundamentos teóricos se abordaron las categorías sustantivas de la inteligencia, inteligencias múltiples y el ajedrez como estrategia lúdica, las cuales se describen a continuación. 


\subsection{La Inteligencia}

El termino inteligencia ha sido definido por diversos autores, quienes la ubican desde el campo psicológico, de la biología, entre otros; de manera que se ha ido fortaleciendo con una firme fundamentación epistemológica y la estructuración de metodologías apropiadas y sensibles a lo más específicamente humano, como es la libertad, la creatividad, la conciencia, los valores y los sentimientos. Para Gardner (1994a): la inteligencia es la capacidad que tiene el ser humano para resolver problemas o elaborar productos relevantes para una cultura o contexto. En este sentido, se concibe la inteligencia como la capacidad general del individuo para comprender y enfrentar su entorno, generando así la combinación de habilidades y factores que se asocian de una manera específica para conformar la aptitud global.

Considerando el ámbito de estudio, desde la educación, cabe referir a Jiménez (citado en Vethencourt, 2017a): quien la conceptualiza más desde la capacidad que tiene el ser humano para relacionar los conocimientos, resolver situaciones problemas en el ámbito personal como en el educativo, abarcando diferentes procesos cognitivos en el constante interactuar consigo mismo, con el otro y el ambiente.

Desde este punto de vista, se resalta que la inteligencia no es permanente, es alterable; en otras palabras, es que cada persona no posee el mismo nivel de inteligencia con el que nace y, la inteligencia no es unitaria, puede manifestarse en diferentes formas; es decir, no hay una sola sino múltiples inteligencias, distintas e independientes. Del mismo modo, la inteligencia se considera como un potencial que puede vincularse con los ámbitos de lo personal, lo colaborativo y lo social. De allí que surge la teoría de las inteligencias múltiples, como contrapeso al paradigma de una sola inteligencia. 


\subsection{Inteligencias múltiples}

Las inteligencias múltiples fueron desarrolladas por Gardner (1994b): fundamentadas en las capacidades del individuo y en la importancia de la inteligencia como la capacidad para resolver problemas cotidianos, generar otros, crear productos y ofrecer servicios dentro de su contexto cultural. Es decir, que todas las personas poseen el potencial para ejercer un conjunto de facultades intelectuales, aunque no las realicen de la misma manera. En este sentido, es de resaltar que la tendencia de resolver problemas tiene que asociarse también al entorno cultural, porque aunque posean estas capacidades en un cierto grado, las personas difieren en el grado de capacidad por la combinación que hace de ellas según las oportunidades que le brinda el contexto.

Por su parte López (2006c): sostiene que "la inteligencia no es vista como algo unitario o que agrupa diferentes capacidades especificas con un destino en nivel de generalidad, sino como un conjunto de inteligencias múltiples, distintos e independientes" (pág. 10). De esta concepción, se habilita la noción de inteligencia como el conjunto de habilidades, talentos y capacidades mentales que posibilitan las herramientas necesarias a todos los seres humanos pueden utilizar para aprender, para resolver problemas y para crear. En cuanto a Prieto y Ballester (2009a): asumen que "las inteligencias son funcionales y que se manifiestan de diferentes maneras en diversos contextos" (pág. 21). En este sentido, la teoría de las inteligencias múltiples proporciona una base sólida sobre la cual identifican y desarrollan un amplio espectro de habilidades en cada persona.

Para presentar la visión epistemológica de los ocho tipos de inteligencias propuesta por Gardner, se toma en cuenta lo expuesto por López (2006d):

Inteligencia Lógica-Matemática: basada en símbolos, permite 
calcular, resolución de problemas, representación de objetos y la distinción de geometrías en el espacio.

Inteligencia Verbal-Lingüística: consiste en la capacidad de pensar en palabras y de utilizar el lenguaje para expresar y apreciar significados complejos. En este sentido, el individuo emplea eficazmente las palabras en forma oral o escrita, para comunicarse a través de diferentes medios.

Inteligencia Visual-Espacial: proporciona la capacidad de pensar, visualizar imágenes internas y externas para crear líneas, formas que representan los objetos reales.

Inteligencia Musical: resulta evidente en los individuos sensibles a la melodía, al ritmo, al tono y a la armonía. De esta manera, se considera que la inteligencia musical es la fundamentada para producir y apreciar las canciones, recordar melodías o simplemente disfrutar de la música.

Inteligencia Corporal-Kinestésica: permite al individuo manipular objetos y perfeccionar las habilidades físicas. Se caracteriza por la habilidad de emplear el cuerpo en diversas formas, de trabajar la motricidad.

Inteligencia Intrapersonal: se refiere a la capacidad de una persona para construir una percepción precisa respecto de si misma y lo usa para la creación de la felicidad personal y social.

Inteligencia Interpersonal: es la capacidad de comprender a los demás e interactuar eficazmente con ellos.

Inteligencia Naturalista: consiste en observar los modelos de la naturaleza, en identificar, clasificar objetos y comprender los sistemas naturales creados por el hombre.

\subsection{Estrategia didáctica}

Como puede apreciarse, son diversas las inteligencias que puede desarrollar cada estudiante, todo depende de las diversas estrategias que el docente propicie para su puesta en práctica por el estudiante, por lo que 
Castellanos y Castro (2017): indican que las estrategias didácticas son las que debe facilitar el docente para el desarrollo de los procesos intelectuales del estudiante, llevándolo a la adquisición de habilidades y destrezas para transferir los conocimientos a la realidad o contexto donde se desenvuelve. En este sentido, se considera al ajedrez como una estrategia didáctica que puede ayudar en el estudiante a desarrollar las diferentes inteligencias.

\subsection{El juego de ajedrez}

El juego para Bello (2007): constituye una actividad voluntaria, que se realiza dentro de ciertos límites fijados de tiempo y lugar, según las reglas establecidas. Para efecto del estudio se enfocará al juego deportivo competitivo, que de acuerdo con el autor, consiste en el ejercicio de inteligencia y acción demostrativo, dirigido a obtener unos resultados individuales o colectivos para alcanzar la meta establecida.

En tal sentido, el ajedrez es visto como un juego deportivo que para Blanco (2007d): es un juego ciencia, debido a la carga intelectual y de razonamiento lógico-matemático que prevalece en la ejecución de una partida y en la resolución de problemas propuesto. En este sentido, se relaciona con la capacidad de observación, del uso de técnicas y métodos para el abordaje y toma de decisiones. Para Gardner (1994c): "probablemente el estudio y la práctica del ajedrez faciliten el estímulo de la inteligencia lógica-matemática, ya que es la habilidad para pensar, calcular y manejar el razonamiento lógico" (pág. 64). Lo cual se relaciona con el pensamiento lógico, la concentración y la memoria visual.

Desde esta perspectiva, se puede decir que, durante una partida de ajedrez, el estudiante se ubica en la posición de resolver un problema, donde observa, compara, clasifica, analiza, organiza ideas, sintetiza, toma decisiones y las ejecuta, además ocurre que tanto jugadores ajedrecistas como estudiantes de otra especialidad, utilizando el tablero y sus figuras pueden 
establecer, explicar relaciones geométricas o espaciales de carácter lógicomatemático.

Así mismo, el ajedrez es una obra de arte, que de acuerdo con Blanco (2007e): "entre dos mentes que necesitan equilibrar dos metas distintas y a veces contradictorias: ganar y producir belleza" (pág. 29). En este escenario, no se puede olvidar que el ajedrez se juega entre dos personas, de cuya confrontación podría generarse la posibilidad de concebir belleza, a la vez que se perciben posiciones y combinaciones de movimientos que generan una fuerte impresión estética tanto en sus productores como en los especialistas y aficionados.

\section{Recorrido Metodológico}

El presente estudio se encuentra fundamentado en el paradigma cualitativo, desde la ideología fenomenológica, debido a que se busca estudiar una realidad, cuya esencia dependerá del modo en que es vivida y percibida por el sujeto, una realidad interna, personal, única y propia de cada ser humano. A partir de este planteamiento, el estudio se abordó desde el método fenomenológico, el cual para Martínez (2011): "la fenomenología es el estudio de los fenómenos tal como son experimentados, vividos y percibidos por el hombre" (pág. 167). En este sentido, la fenomenología surge como una necesidad de explicar la esencia de las cosas, es decir, es un método que observa y explica la vivencia para conocerla con exactitud y, de esta forma, encontrar la verdad de los fenómenos. El recorrido metodológico seguido fue:

Etapa previa, que permitió clarificar los presupuestos que determinaron el curso de la investigación, visitando a diferentes instituciones donde se practicaba el ajedrez, seleccionando la Unidad Educativa de Talentos Deportivos, del Municipio Valera, estado Trujillo.

Etapa descriptiva, el objetivo de esta etapa fue lograr una descripción del fenómeno en estudio, el permiso y autorización para el estudio, así como 
la elección de la técnica de la entrevista para obtener la información, realizando en un primer momento un protocolo de entrada, socializado y depurado para hacer más efectivo el conversatorio, siendo grabado, previo permiso solicitado. Permitiendo obtener información para estructurar el guion de la entrevista o conversatorio en función del propósito de estudio.

Etapa estructural, consistió en el estudio de las descripciones contenidas en el guion, permitiendo realizar la entrevista o conversatorio cara a cara para obtener la información necesaria, la misma fue grabada en una videograbadora, y las anotaciones en un cuaderno de nota que permitió registrar algunas apreciaciones de las investigadoras.

Etapa discusión de los resultados, se sistematizó la información por actor clave, a quienes se les asignó un código para resguardar su privacidad, luego extraer las categorías emergidas y triangular desde las voces de los actores sociales, confrontar o sustentar desde los teóricos y la interpretación de las investigadoras a partir de la realidad percibida.

Actores clave, según Tapella (citado en Vethencourt, 2017b): son usualmente considerados como aquellos que pueden influenciar significativamente (positiva o negativamente de una intervención) o son muy importantes para que una situación se manifieste de determinada forma. Es decir, son aquellas personas cuya participación es imprescindible para el logro de propósitos, objetivos y metas del estudio. Para efecto de la investigación, los actores claves fueron seis (6) estudiantes de la Unidad Educativa de Talento Deportivo Valera estado Trujillo donde se realizó el estudio, quienes fueron seleccionados de acuerdo con los siguientes criterios:

- Ser estudiante de la Institución.

- Ser jugador de la disciplina de ajedrez.

- Índice académico de bueno a excelente. 


\section{Resultados}

Entre las categorías emergidas sobre las inteligencias manifestadas por los estudiantes a través del ajedrez, se apreció la lógica-matemática, la lingüística, la visual-espacial y la intrapersonal, que se describen a continuación, fundamentadas o confrontadas desde los teóricos y las investigadoras.

4.1. La inteligencia lógica-matemática, fue una de las categorías que prevaleció desde el discurso de los estudiantes, la cual se refleja en las subcategorías surgidas, tales como: resolver problemas, razonar, calcular, y relación matemática, tal como se aprecian a continuación:

Resolver problemas, de acuerdo con FR expresó "los estudios se me hacen mucho más fácil, gracias a la práctica del ajedrez, debido a que durante los entrenamientos resolvemos problemas", de igual forma, YG manifestó "me va muy bien en los estudios, me gusta cada clase y se me hace fácil de entenderla, porque como práctico el ajedrez, pues aquí se da mucho la matemática y no me cuesta como en ninguna otra materia". De igual manera, OM expresa "el ajedrez es un juego de concentración y en la práctica me ayuda a la solución de problemas, por ejemplo, la matemática se basa en problemas entonces aquí nos ayuda a solucionarlos y es más fácil entenderlos, captar más rápido estando en la clase".

Como puede apreciarse, a través del ajedrez, el estudiante deportista, desarrolla su habilidad para resolver problemas, a la vez que le permite mantener una mayor concentración, relacionando sus conocimientos con las actividades escolares que desarrolla en la matemática. Lo cual para Antunes (2001a): la lógica-matemática es muy importante, ya que permite resolver incluso problemas a los que nunca se ha enfrentado el ser humano utilizando solamente su inteligencia. Lo cual le lleva a tomar decisiones a la hora de realizar una jugada.

Calcular, fue otra de las habilidades que manifestaron los participantes 
desde el juego de ajedrez, como lo expresa SV "Me va bien en los estudios porque el ajedrez me ayuda a pensar y analizar cuando hago los cálculos, pues me permite sumar, restar y hasta multiplicar". Del mismo modo, JP "Se me hace fácil la matemática porque cuando entreno ajedrez vemos problemas de combinaciones y tácticas, eso me ayuda a solucionar problemas de cálculos matemáticos".

Al respecto, Antunes (2001b): indica que aprender matemáticas, física o química "es muy difícil", así se expresan la mayoría de los estudiantes de todos los niveles educativos; lo que se contradice con lo señalado por los actores claves de esta investigación, quienes expresaron que con la práctica del ajedrez se les hace más fácil entender las matemáticas, pues realizan las operaciones que aplican durante el juego de ajedrez.

Razonar, sub-categoría que emerge desde las voces de los estudiantes deportistas, al referir SV "El ajedrez me ayuda a razonar la manera en que debo hacer la jugada". Razonar que lleva implícito el proceso de calcular y que le permite al participante, resolver los problemas de manera efectiva. En este caso, el razonar es parte esencial en el desarrollo de la inteligencia lógicamatemática propuesta por Gardner (1994d): "en su teoría de las inteligencias múltiples que define la inteligencia lógica-matemática como la capacidad de utilizar números de manera efectiva y de razonar adecuadamente empleando el pensamiento lógico-matemático".

Relación matemática, así en la voz de YG "Yo digo que el ajedrez nos ayuda en todas las materias porque es un deporte de mucha concentración y esto hace que prestemos atención a las clases". Por su parte JP dijo, "Yo relaciono el ajedrez con la matemática porque cuando corono peones, se pueden convertir en otras piezas de mayor validez de puntuación, es decir, se multiplican las piezas como por ejemplo el peón que vale un punto al coronar lo cambio por una dama que vale diez puntos y múltiplo 1 por 10. También la resta y las suma: cuando le capturo varias piezas a mi oponente le resto y la 
sumo a la vez si voy ganando o perdiendo tanto en piezas como su valor de puntos".

Por su parte, ES dijo "Yo relaciono el ajedrez con la matemática porque se usan los números", de igual manera, YG manifestó "Yo lo relaciono con la matemática y la geometría, porque es la que se utiliza en todo ya que se suman, se restan y se multiplican números, así como también las casillas blancas y negras del ajedrez son pares y el tablero está dividido en filas representadas en números del uno al ocho". Para FR "El ajedrez si ayuda como estrategia para los estudios porque sirve como herramienta para el desarrollo mental. Asimismo, nos ayuda en todas las materias como en la física, la química, la matemática y comercio porque es donde se dan los números a diferencia de las demás materias".

Considerando las expresiones señaladas, Blanco (2007f): hace referencia al relacionar con uno de los objetivos del constructivismo, el cual es la construcción del conocimiento. Por lo que plantea, que el tema de la lógicamatemática, se presta para que el estudiante pueda realizar las relaciones entre las distintas proporciones, creando nuevas formas de resolver problemas en distintas ramas como en: matemáticas, física, química, ciencias sociales, como en el ajedrez y, por supuesto, de la vida real.

Desde las subcategorías emergidas en lo que corresponde a la lógicamatemática, López (2006e): señala que ésta inteligencia "ve la habilidad de utilizar números para calcular y describir, utilizar conceptos matemáticos para hacer conjeturas, aplicar matemáticas en la vida diaria personal, y resolver problemas en diseño y en modelado" (pág. 15). En este sentido, relacionando estos planteamientos con el ajedrez, un jugador de este deporte se encuentra dotado de la inteligencia lógica-matemática, específicamente cuando se habla del proceso de resolver problemas, calcular y razonar, llevándolo a tomar decisiones desde las diversas variantes que se le presentan en un juego.

Inteligencia que a la vez los lleva al desarrollo mental como lo 
manifestaron los estudiantes, el cual para Gutiérrez (2005), es la adquisición de la capacidad motora y de la habilidad manual, de la facultad de la palabra, de la inteligencia, del estudio y de la capacidad de resolver los propios problemas y de ambientarse en el medio social. En este sentido, a través de la práctica del ajedrez, los estudiantes atletas ponen en práctica la inteligencia lógica-matemática, para resolver problemas, razonar y calcular en una partida de ajedrez, permitiéndoles así un buen desarrollo mental.

4.2. Inteligencia Lingüística-Verbal, fue otra de las categorías surgidas en los actores socioeducativos participes en este estudio, que de acuerdo con Gardner (1994e): está relacionada con la capacidad de usar palabras de manera efectiva al escribirlas o al hablarlas. En lo que FR manifestó "El ajedrez está relacionado con las letras, a la vez con lenguaje y literatura, ya que al jugar se anotan las partidas jugadas en un lenguaje ajedrecístico".

Así mismo, OM dijo "El ajedrez en la parte académica me ayuda en lenguaje y literatura específicamente cuando se hace análisis y síntesis sobre la materia concentrándose como en el ajedrez que se necesita mucha concentración cuando vas a leer y estudiar los libros de aperturas que uno juega", del mismo modo, para JP indicó: "El ajedrez me ayuda como estrategia en lenguaje y literatura ya que he mejorado mucho en la lectura, porque en el ajedrez se tiene que leer muchos libros sobre aperturas $y$, en la escritura porque cuando se juega una partida se tiene que anotar en un lenguaje ajedrecístico".

Como puede apreciarse, los estudiantes refieren que el ajedrez les ayuda en el área de lengua y literatura, en el proceso de lectura y escritura, en el análisis y síntesis que deben realizar para prepararse y realizar las jugadas o torneos de ajedrez, Cabe retomar a Gardner (citado en Blanco; 2007g): cuando planteó que las prácticas del ajedrez podrían estar asociadas muy íntimamente con la inteligencia lingüística. Desde este punto de vista, se puede decir que la categoría lingüística-verbal se encuentra íntimamente 
ligada con el ajedrez, debido a que relacionan la materia de lenguaje y literatura con el ajedrez, puesto que en estos ambientes se encuentran ricos lingüísticamente.

Asimismo, se brinda un ambiente donde ellos se sienten lo suficientemente seguros para expresar, explicar sus ideas, comparten sus opiniones o anécdotas de como por ejemplo cuando jugaron mal o bien en una partida y de esta manera contribuyen al aumento de su autoestima. Acosta, Rojas y Medina (2011): plantean que la inteligencia lingüística-verbal es la capacidad de pensar en palabras y de utilizar lenguaje para comunicarse, comprender, expresar y apreciar significados complejos. Es de resaltar que dentro del juego de ajedrez, existe un lenguaje ajedrecístico, el cual es la anotación del sistema algebraico, esto es cuando se está jugando una partida se va anotando las jugadas, tanto de ellos mismos como las de su oponente.

4.3. Inteligencia Visual-Espacial, siendo otra de las inteligencias percibidas por los estudiantes atletas, pues YG señaló "El ajedrez me ayuda en artística porque allí representan figuras, dibujos, colores y formas de las piezas sobre el tablero". Desde esta perspectiva, el autor Prieto y Ballester (2009b): exponen que la inteligencia visual-espacial es la que incluye la sensibilidad del color, la línea, la forma, el espacio y las relaciones que existen entre estos elementos. Incluye la capacidad de visualizar, representar de manera gráfica ideas visuales o espaciales.

Por su parte, Blanco (2007h): plantea que "la representación espacial en el juego del ajedrez se desarrolla con figuras de diferentes colores que se desplazan e interactúan sobre la superficie plana llamada tablero" (pág. 62). Así mismo el autor plantea, que le permite al jugador poner en práctica su capacidad de percepción global (holística) de situaciones de razonamiento abstracto y orientación espacial, de imaginación, creación e inventiva.

Desde este punto de vista, el jugador de ajedrez pone en práctica la creatividad para trasladar mentalmente las piezas de un sitio a otro, el jugador 
visualiza la posición y el color de las piezas sobre el tablero en un espacio en el presente, luego analiza esa posición con sus posibles jugadas de variantes, sub-variantes que se pueden dar para así lograr realizar las mejores jugadas trasladando espacialmente la posición en el presente hacia al futuro, con el fin de realizar las mejores jugadas, y así ganar la partida.

4.4. Inteligencia Intrapersonal, que emergió en la voz de SV, quien dijo: "Me siento bien, emocionado y concentrado pensando en la jugada que voy hacer y en las combinaciones". De la misma manera, para OM manifestó: "Bueno cuando juego el ajedrez me siento emocionada, pero al mismo tiempo nerviosa, ya que hay que comenzar a jugar una nueva partida desde el inicio, esta se alarga y uno nunca sabe lo que pueda llegar a ocurrir".

Desde estas expresiones, se ven reflejadas las emociones como parte elemental del ser humano, por lo que López (2006f): indica que "la inteligencia emocional tiene que ver con nosotros y con nuestro mundo interior: Capacidades inherentes a las que recurrimos para comprendernos a nosotros mismos y a otras personas; para imaginar planificar, y resolver problemas especialmente los emocionales" (pág. 95). De igual forma la Serrano (2003): señala que la inteligencia intrapersonal es el conocimiento y la habilidad para adaptar las propias maneras de actuar a partir del conocimiento. Incluye tener una imagen precisa de uno mismo, tener conciencia de los estados de ánimos interiores, las intenciones, las motivaciones, los temperamentos y los deseos.

En este sentido se puede decir que, la inteligencia intrapersonal es la que tiene acceso a los sentimientos propios y a la habilidad para comprender las emociones intimas, a los conocimientos de las fortalezas y debilidades propias, esta premisa tiene mucha relación con la de un ajedrecista en cuanto a la comprensión de sus emociones, debido a que éste se encuentra preparado para comprender cuando pierde o gana una partida, asimismo, conoce cuando tiene una debilidad y fortaleza. Lo que le lleva a tener un dominio personal al momento de jugar el ajedrez. 
Se pudo apreciar que el ajedrecista tiene la habilidad de meditar cuando está jugando una partida, tiene una disciplina personal en cuanto a su vida y a sus entrenamientos, conserva la compostura con su oponente cuando gana 0 pierde un juego. Postura que la ponen en práctica durante el proceso educativo.

\section{A manera de cierre}

Desde el estudio realizado y considerando el propósito de investigación, se puede señalar que los estudiantes que practican el ajedrez tienen la facilidad para desarrollar las inteligencias Lógica-matemática, la cual les ayuda a resolver problemas, a razonar y a calcular cuando están jugando una partida de ajedrez, desarrollando procesos mentales que transfieren en las demás áreas del saber. Así mismo, les permite desarrollar la capacidad de usar palabras de manera efectiva al escribir o al hablar, cuando anotan una partida con el sistema algebraico y la analizan con sus oponentes; fortaleciendo así la lectura y escritura.

Por otra parte, desarrollan la capacidad de representación espacial, en el juego del ajedrez, llevándolos a percibir de forma holística las jugadas, analizando cada evento desde las diversas jugadas que puede realizar. De igual manera, tiene la capacidad de relacionar las figuras y los colores al realizar los desplazamientos en el tablero. Así mismo, la inteligencia intrapersonal, como la capacidad para acceder a sus propios pensamientos y sentimientos en cada jugada.

Desde los hallazgos develados a partir de las voces de los actores claves del estudio, se hace necesario recomendar a los docentes de las diversas instituciones educativas, poner en práctica la estrategia del ajedrez como herramienta para fortalecer las inteligencias de sus estudiantes, tanto en la práctica del deporte como en su rendimiento académico. Que se preparen y busquen personas que le orienten en cómo aprender y enseñar el ajedrez 
en los espacios de aprendizaje.

Solicitar entre los estudiantes los que dominan el ajedrez para que se sientan motivados a enseñar-aprender-jugar y relacionar con los demás, contenidos académicos. Así mismo, conformar con sus estudiantes un club o centro de ajedrez, que los lleve a relacionar, a recrearse en la medida que aprende a resolver problemas, a compartir y competir, llevándolos a tomar decisiones en cada jugada, a demostrar sus conocimientos, habilidades y experiencia desde la preparación y entrenamiento. Pues se trata de ayudar a desarrollar la capacidad intelectual, como la atención, el razonamiento, el análisis, la síntesis, la creatividad, entre otros.

\section{Referencias}

Acosta, J., Rojas, A., \& Medina, A. (2011). Inteligencias Múltiples en el Aula de Clase. Caracas, Venezuela: Universidad Pedagógica Experimental Libertador.

Altuve, S. (2015). Ajedrez como Estrategia Ecológica dirigido al Docente para el desarrollo de las Inteligencias Múltiples en los Estudiantes. Trabajo Especial de Grado de Maestría. Rubio, Venezuela: Universidad Pedagógica Experimental Libertador.

Antunes, C. $(2001 a, b)$. Estimular las inteligencias múltiples: qué son, cómo se manifiestan, cómo funcionan. (2da. Edición). Madrid, España: Narcea.

Bello, A. (2007). El deporte. El juego de la competencia. Venezuela: Panapo. Blanco, U. (2007a,b,c,d,e,f,g,h). ¿Por qué el Ajedrez en las escuelas?. Caracas, Venezuela: Ediciones CO-BO.

Blanco, J., Fernández, J., Mayor, R., \& Martos, M. (2010). La Educación Emocional en el ajedrez. Propuestas para aplicar en los centros educativos. España: Associació Paretana D'escacs. Recuperado de: http://www.paretana.com/zip/articulos/La\%20educaci\%F3n\%20emocio 


\section{nal\%20en\%20el\%20ajedrez.pdf}

Castellanos, E., \& Castro, J. (2017). Estrategias didácticas para mejorar la lectura y la escritura. Revista Scientific, 2(6), 74-91. Recuperado de: https://doi.org/10.29394/scientific.issn.2542-2987.2017.2.6.4.74-91

Gardner, H. (1994a,b,c,d,e,f). Inteligencias Múltiples. La teoría en la práctica. Barcelona, España: Paidós.

Gutiérrez, F. (2005). Teorías del Desarrollo Cognitivo. Buenos Aires: McGraw-Hill.

López, A. (2006a,b,c,d,e,f). Inteligencias Múltiples Cómo Descubrirlas y Desarrollarlas. 1era. Edición. Lima, Perú: Ediciones Mirbet.

Martínez, M. (2011). Comportamiento Humano, Nuevos Métodos de Investigación. México: Trillas.

Pérez, M. (2012). Inteligencia y ajedrez: estructura y contexto de una relación familiar. España: Punto Rojo Libros S.L. ISBN: 978.84-1556180-4, págs. 350.

Prieto, M., \& Ballester, P. (2009a,b). Las Inteligencias Múltiples. Diferentes formas de enseñar y aprender. Madrid, España: Pirámide.

Serrano, A. (2003). Inteligencias Múltiples y Estimulación Tempranas. Guía para educadores, padres y maestros. México: Trillas.

Vethencourt, M. (2017a,b). Uso del ajedrez como estrategia en el desarrollo de la inteligencia del estudiante. Tesis Doctoral en Educación. Cabimas, Venezuela: UNERMB. 


\section{Yuraima Margelis Matos De Rojas \\ e-mail: yuraimatos01@gmail.com}

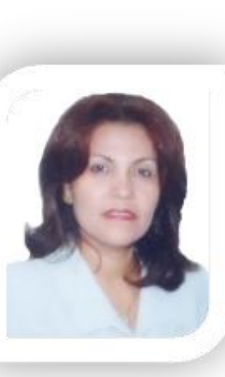

Nacida en Venezuela. Licenciada en Educación Integral, Mención Matemáticas, Magister en Ciencias de la Educación. Mención Investigación Docencia. Magister en Tecnología y Diseño educativo, Magister en Educación Robinsoniana, Dra. En Ciencias de la Educación. Docente investigador en la Universidad Nacional Experimental Simón Rodríguez, (UNESR) Núcleo Valera, Edo. Trujillo (Jubilada). Coordinadora de la Línea de investigación: Investigadores en acción Social (IAS), Facilitadora en cursos de metodología, Seminario de investigación, Valores, Servicio Comunitario, Proyecto de Investigación, administración de la educación Básica, Ética y valores en la investigación, gerencia educativa, entre otros cursos, en las Universidades: Simón Rodríguez, Valle del Momboy, Rafael María Baralt (Especialidad, Maestría y Doctorado). Jurado evaluador y tutora de Trabajo Especial de Grado y Tesis, Evaluadora de trabajos de ascenso, artículos arbitrados en Revistas nacional e internacional. Actualmente miembro del PEII, NIVEL B. 


\section{Maryelin Lorena Vethencourt Godoy}

e-mail: mayerajedrez@gmail.com

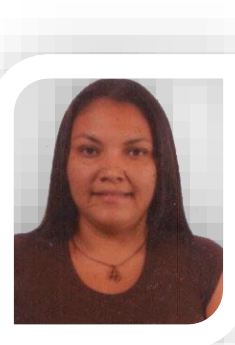

Nacida en Trujillo, Venezuela. Licenciada en Educación Integral, Área Matemática, Magister en Administración de la Educación Básica, Estudiante del Doctorado en Educación de la Universidad Nacional Experimental "Rafael María Baralt". Maestra Nacional de Ajedrez. Laboro actualmente en U.E. De Talentos Deportivos del Estado Trujillo. Profesora de la UNEFA Núcleo Zulia, Presidente de la Asociación Trujillana de Ajedrez y entrenadora del Estado Trujillo, Facilitadora de talleres y arbitraje de Ajedrez. 


\section{Magda Lisbeth Peña Briceño}

e-mail: bmagdaunica@hotmail.com

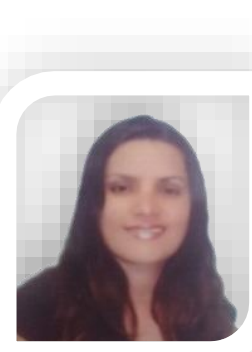

Nacida en Trujillo, Venezuela. Licenciada en Educación Integral, Licenciada en Educación Preescolar, Magister en Administración de la Educación Básica. Doctorante de la Universidad Experimental "Rafael María Baralt". Laboro actualmente en la U.E. José Gregorio Hernández. 


\section{Magda Violeta Briceño \\ e-mail: bmagda@hotmail.com.ar}

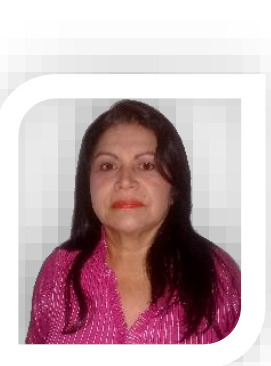

Nacida en Trujillo, Venezuela. Licenciada Mención Biología, Licenciada en Educación Preescolar, Magister en Administración de la Educación Básica. Doctorante de la Universidad Experimental "Rafael María Baralt". Laboro actualmente como directora en la U.E. José Gregorio Hernández. 